\title{
Mass outflow constraints in active nuclei and quasars from X-ray spectroscopy
}

\author{
Tahir Yaqoob $^{1}$ \\ ${ }^{1}$ Department of Physics and Astronomy, Johns Hopkins University, 3400 N. Charles St., \\ Baltimore, MD 21218, USA
}

\begin{abstract}
Absorption in the X-ray spectra of active galactic nuclei from outflowing gas can be modeled to yield critical physical information on the outflows. The outflow rate of mass ejected back into the ISM of the host galaxy and the resulting feedback could potentially have an impact on evolution. We give a brief overview of the current observational constraints on the outflows that should be taken into account by models of evolution and feedback.
\end{abstract}

Keywords. galaxies: active - ISM: jets and outflows - X-rays: galaxies

\section{Photoionized Outflows Observed with X-ray Spectrometers}

The advent of high-resolution X-ray grating spectrometers aboard Chandra and XMMNewton have now lead to detailed measurements of key physical parameters of photoionized outflows from galactic nuclei (e.g. Blustin et al. 2005; McKernan, Yaqoob, \& Reynolds 2007, and references therein). A common feature in the X-ray spectra of Seyfert galaxies is discrete absorption lines that are typically blueshifted by $v \sim$ $100-1000 \mathrm{~km} \mathrm{~s}^{-1}$, and are usually unresolved (FWHM $<300 \mathrm{~km} \mathrm{~s}^{-1}$ at the best spectral resolution). In some quasars, very high velocity $\left(\sim 20,000-60,000 \mathrm{~km} \mathrm{~s}^{-1}\right)$, high ionization outflows have been reported using X-ray CCD detectors (e.g. Reeves et al. 2003, and references therein). The mass outflow rate can be written

$$
\dot{M}_{\text {out }}=1.658 x y\left(\frac{\Delta \Omega}{4 \pi}\right) \frac{L_{\text {ion }, 44}}{\xi_{1000}} v_{500} f \quad M_{\odot} \mathrm{yr}^{-1}
$$

where $\Delta \Omega /(4 \pi)$ is the solid angle subtended at the ionizing source, which has a 11000 Rydberg luminosity of $L_{\text {ion, } 44}$ (in units of $10^{44}$ ergs s$^{-1}$ ), $\xi \equiv 1000 \xi_{1000}$ is the ionization parameter, and $v\left(\mathrm{~km} \mathrm{~s}^{-1}\right) \equiv 500 v_{500}$ is the outflow velocity. If $n_{e}\left(\mathrm{~cm}^{-3}\right)$ is the electron density, and $R(\mathrm{~cm})$ is the distance of the innermost part of the outflow from the ionizing radiation, $\xi \equiv L_{\text {ion }} /\left(n_{e} R^{2}\right)$. A critical quantity is $f$, the volume filling factor, which constitutes the largest uncertainty since it cannot be directly measured yet. If the outflow uniformly fills the volume, $f \sim 1$, but if it is in the form of a "spray" or "mist", $f$ could be orders of magnitude less than unity (or the outflow could be clumpy, consisting of clouds, resulting in $f<1$ ). Finally, $x$ is the mean number of Hydrogen atoms per electron and $y$ is the mean atomic mass per Hydrogen atom. For a gas consisting of only $\mathrm{H}$ and $\mathrm{He}$, in which $\mathrm{He}$ is $10 \%$ abundant by number, $y=1.3$ and $x=9 / 11$, so $x y=1.0636$. Given the uncertainty in $f, \dot{M}_{\max }=1.76 L_{\mathrm{ion}, 44} v_{500} / \xi_{1000} M_{\odot} \mathrm{yr}^{-1}$ can be interpreted as an upper limit on $\dot{M}_{\text {out }}$. For a merged sample of 13 active galaxies (AGNs) in McKernan et al. (2007) and Blustin et al. (2005) in which photoionized outflows were detected, this upper limit lies in the range $\sim 0.3-3000 M_{\odot} \mathrm{yr}^{-1}$. If the filling factor were a few percent and $\Omega /(4 \pi) \sim 0.1$ then $\dot{M}_{\max } \sim 10^{-3}$ to $\sim 10 M_{\odot} \mathrm{yr}^{-1}$. For the few quasars with relativistic outflows, $L_{\text {ion }, 44} v_{500} / \xi_{1000}$ may be as much as $\sim 10^{4}$ larger than for Seyfert galaxies. Note that Blustin et al. (2005) estimate $f$ up to $\sim 8 \%$, assuming that 

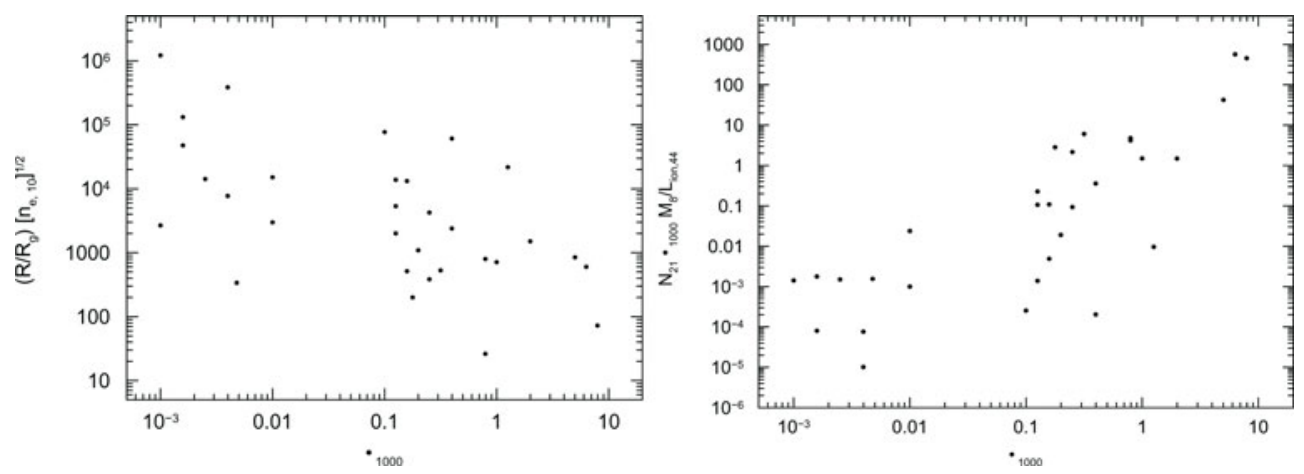

Figure 1. (a) (Left): Distance factor of some AGN outflows. (b) (Right): Gravitational broadening factor. Shown are results for 31 ionized outflow components from 13 sources.

the momentum in the outflow is equal to the incident radiation momentum. However, this need not be the case and the assumption leads these authors, for some sources, to derive upper limits on the distance of the outflow from the radiating source that are less than the lower limits, which is not possible.

The distance of the outflow from the ionizing source, in gravitational radii, is $R / R_{\mathrm{g}}=$ $213.35 M_{8}^{-1}\left[L_{\mathrm{ion}, 44}\right]^{\frac{1}{2}}\left[n_{\mathrm{e}, 10} \xi_{1000}\right]^{-\frac{1}{2}}$ where $M \equiv 10^{8} M_{8} M_{\odot}$ is the black hole mass and $n_{\mathrm{e}}=$ $10^{10} n_{\mathrm{e}, 10}$. From time-averaged X-ray spectra, $n_{\mathrm{e}}$ is not directly measurable so Figure 1(a) shows $\left(R / R_{\mathrm{g}}\right) \sqrt{n_{\mathrm{e}, 10}}$ (individual sources may contain multiple outflow components).

\section{Gravitational Line Broadening Constraints}

An interesting lower limit on $f$ is obtained if $\left(R / R_{\mathrm{g}}\right)<[c / \mathrm{FWHM}]$ where FWHM is the upper limit on the width of the unresolved X-ray absorption lines. Since the radial extent of the outflow gives rise to gravitational broadening, which cannot broaden the lines more than the observed upper limit, we get

$$
f \geqslant 1.8 \times 10^{-4}\left[\frac{300 \mathrm{~km} \mathrm{~s}^{-1}}{\mathrm{FWHM}}\right] \frac{N_{21} \xi_{1000} M_{8}}{L_{\mathrm{ion}, 44}}
$$

where $N_{21}$ is the line-of-sight column density (in units of $10^{21} \mathrm{~cm}^{-2}$ ), which may lie in the range $N_{21} \sim 0.1-100$. Figure 1 (b) shows the range in the parameter that determines the importance of gravitational broadening, $\left(N_{21} \xi_{1000} M_{8}\right) / L_{\text {ion }, 44}$, for the sample of 13 AGN mentioned above. The limit holds only if $\left(R / R_{\mathrm{g}}\right)<[c / \mathrm{FWHM}]$ (otherwise gravitational broadening is never enough to attain the observed upper limit on line width). This implies a lower limit on the mass outflow rate for such compact outflows if they originate at a distance greater than the marginally stable orbit around a Schwarzschild black hole: $\dot{M}_{\text {min }}=5.5 \times 10^{-5} x y[\Delta \Omega /(4 \pi)] N_{21} M_{8} v_{500} M_{\odot} \mathrm{yr}^{-1}$. For the sample described above, the quantity $N_{21} M_{8} v_{500}$ spans a very wide range, $\sim 6 \times 10^{-5}$ to $\sim 80$.

\section{Acknowledgements}

The author thanks Barry McKernan and Chris Reynolds.

\section{References}

Blustin, A., et al. 2005, A\& $A, 431,111$

McKernan, B., Yaqoob, T. \& Reynolds, C. S. 2007, MNRAS, 379, 1359

Reeves, J. N., O’Brien, P. T. \& Ward, M. J. 2003 ApJ, 593, L65 


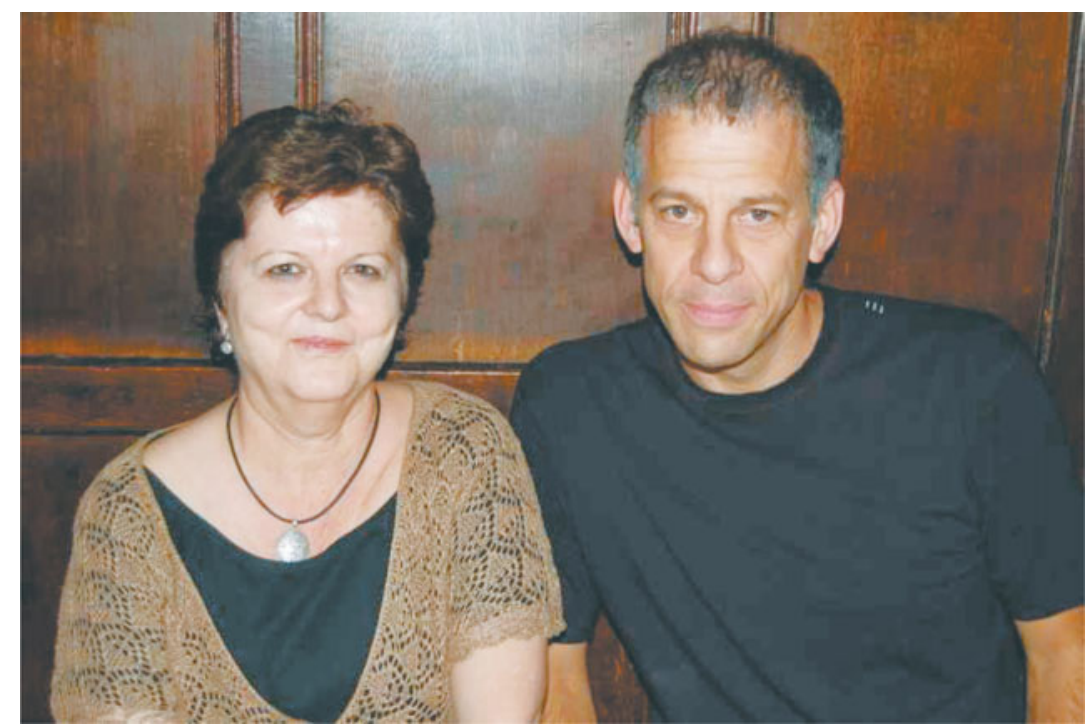

Figure 2. Rosa Domínguez-Tenreiro and Stéphane Courteau during the symposium dinner.

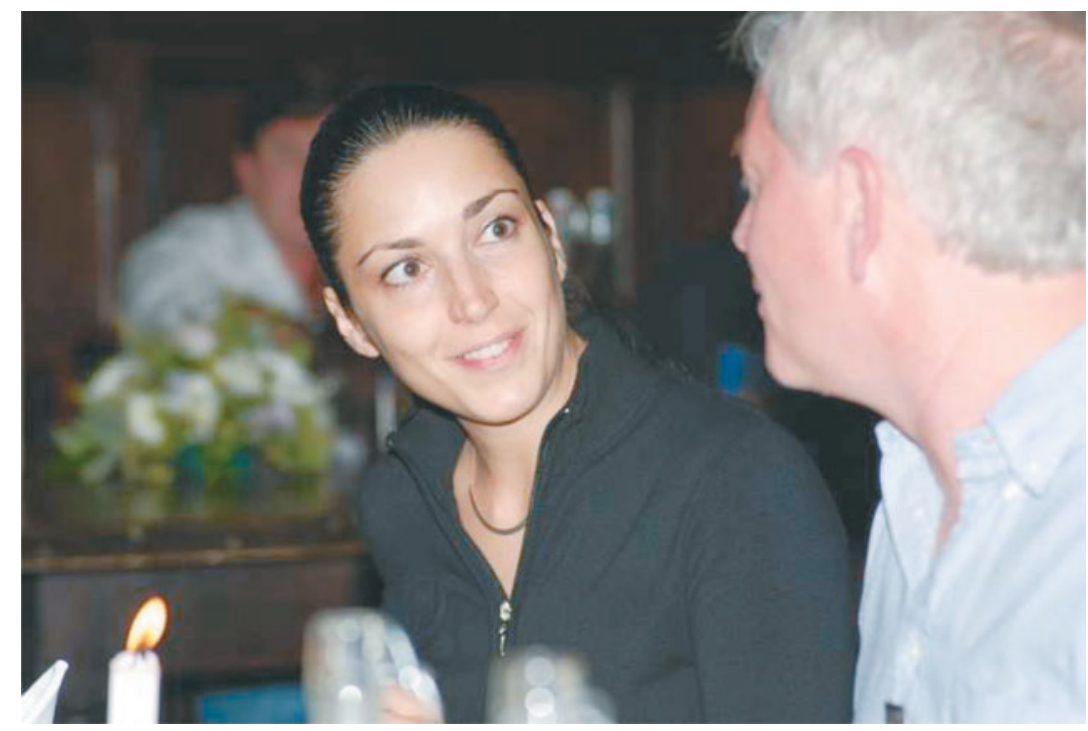

Figure 3. Elena Dalla Bontà and Christopher O'Dea during the symposium dinner. 


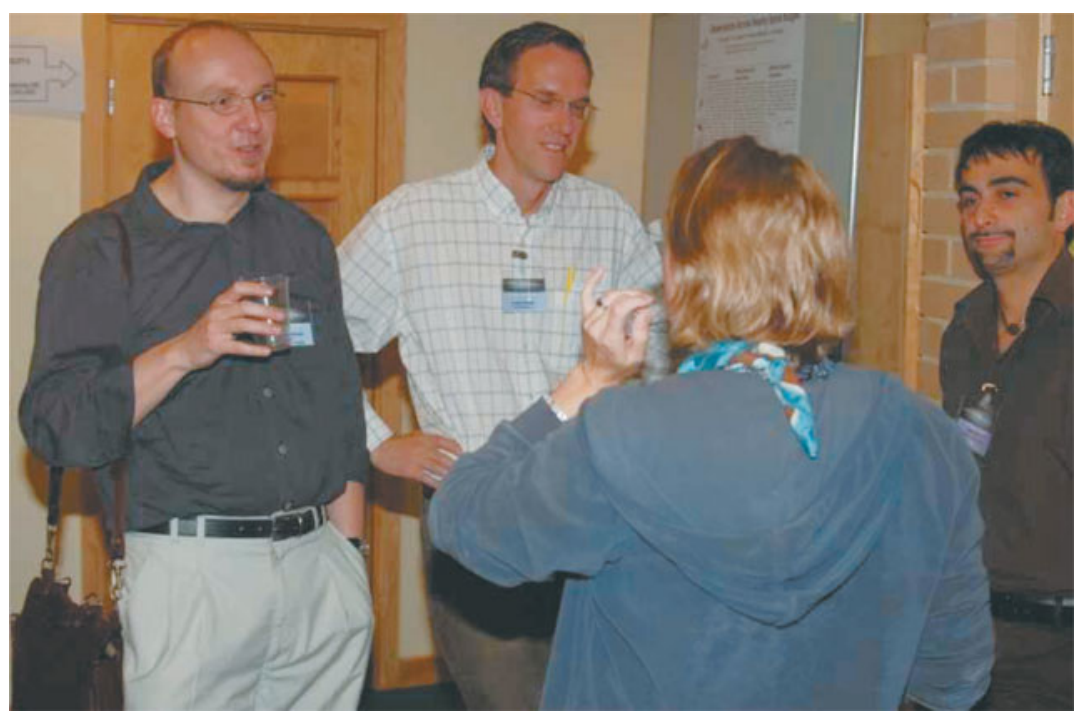

Figure 4. Witold Maciejewski, Joseph Shields, Eva Schinnerer and LOC member Marc Sarzi discussing during a coffee break.

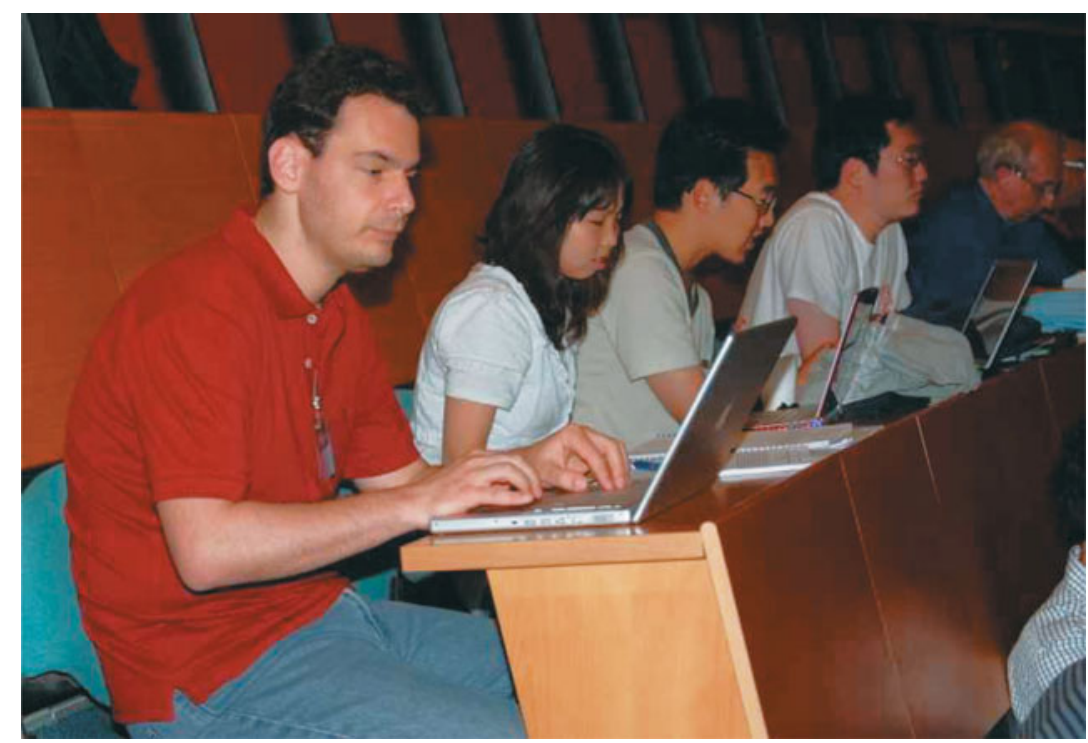

Figure 5. Niv Drory, Hyunjin Jeong, Joo Heon Yoon, Jae-Woo Kim and John Lucey hard at work during the symposium. 


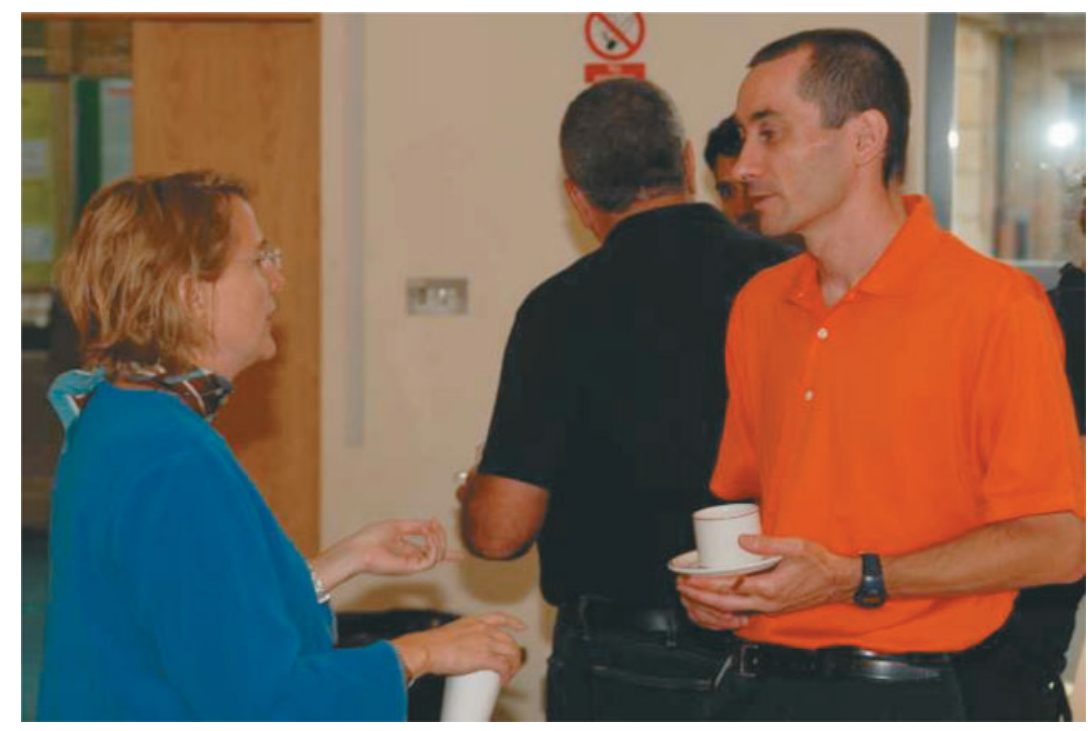

Figure 6. Eva Schinnerer and Roeland van der Marel discussing during a coffee break. Avishai Dekel is visible in the background.

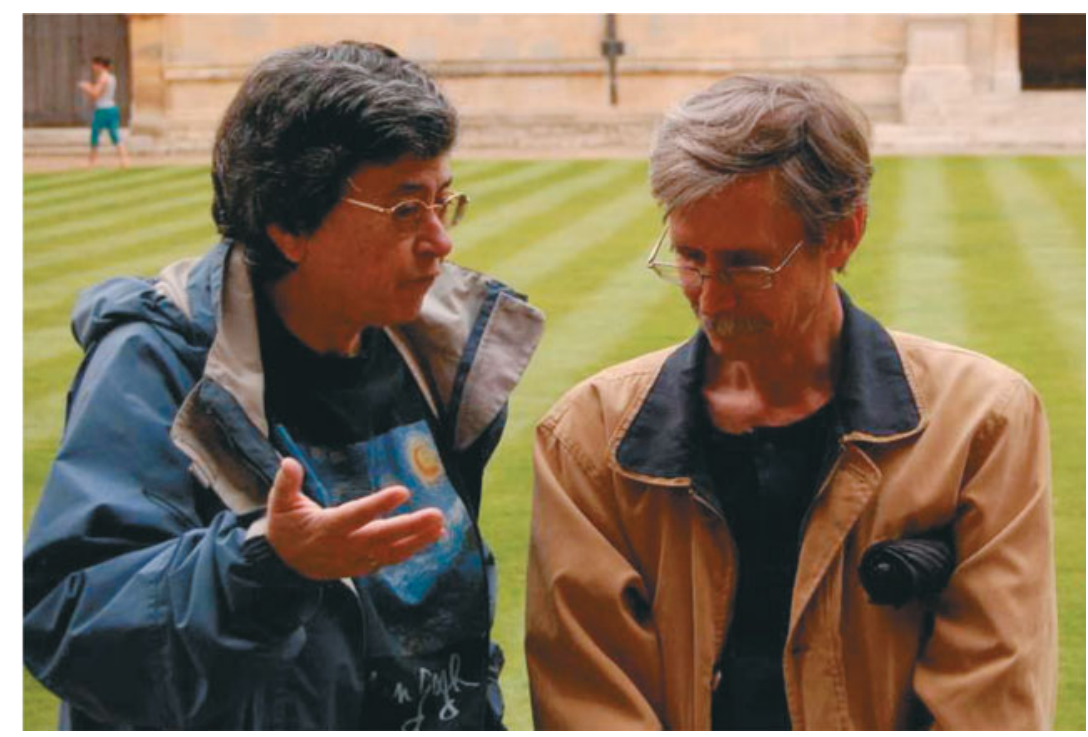

Figure 7. SOC member Lia Athanassoula and Anatoly Klypin discussing in the front quad of Wadham College. 


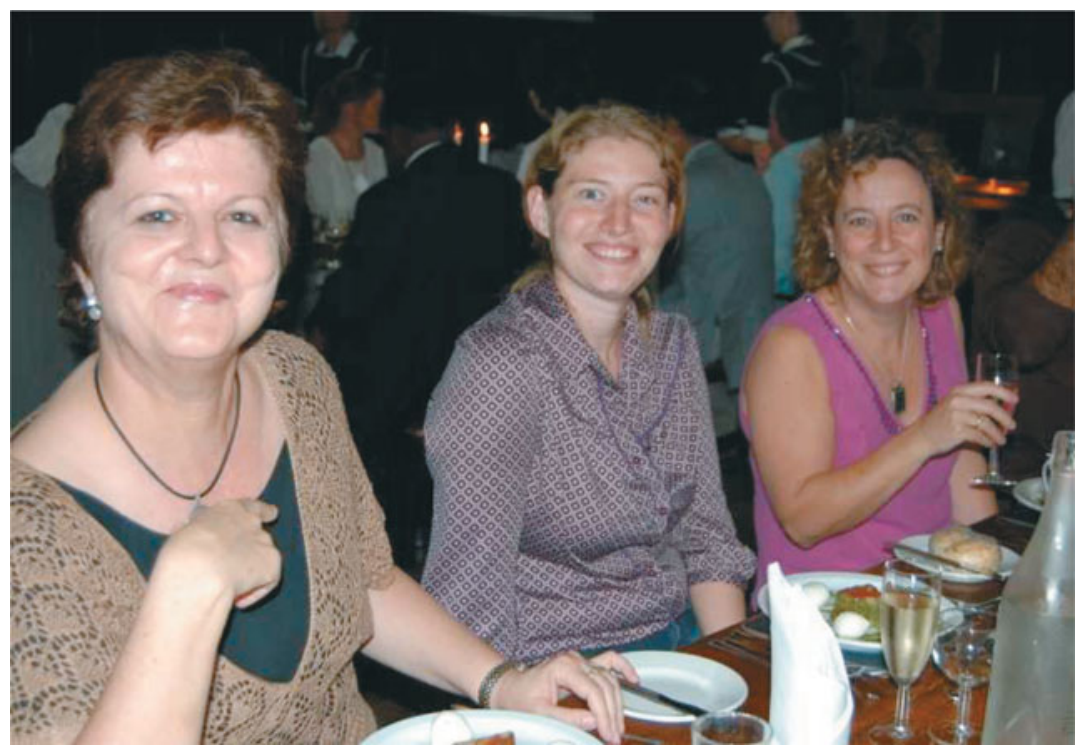

Figure 8. Rosa Domínguez-Tenreiro, Inma Martinez-Valpuesta and Mercedes Mollá during the symposium dinner.

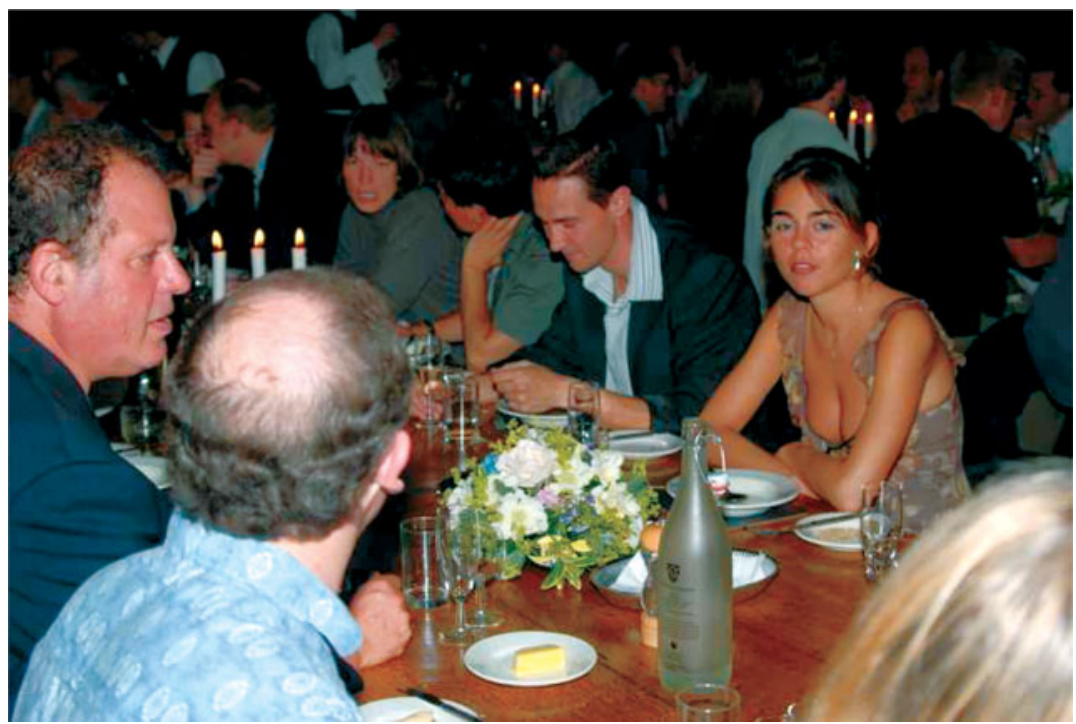

Figure 9. Michael Rich and Bryan Miller (front), and from right to left Katia Ganda, Sebastian Haan, Jong-Hak Woo, Lisa Young and Witold Maciejewski during the symposium dinner. 